\title{
Effects of knee joint angle on global and local strains within human triceps surae muscle: MRI analysis indicating in vivo myofascial force transmission between synergistic muscles
}

\author{
Peter A. Huijing · Alper Yaman • Cengizhan Ozturk • \\ Can A. Yucesoy
}

Received: 11 November 2010/ Accepted: 5 August 2011/Published online: 13 September 2011

(C) The Author(s) 2011. This article is published with open access at Springerlink.com

\begin{abstract}
Purpose Mechanical interactions between muscles have been shown for in situ conditions. In vivo data for humans is unavailable. Global and local length changes of calf muscles were studied to test the hypothesis that local strains may occur also within muscle for which global strain equals zero. Methods For determination of globally induced strain in $\mathrm{m}$. gastrocnemius in dissected human cadavers several knee joint angles were imposed, while keeping ankle joint angle constant and measuring its muscle-tendon complex length changes. In vivo local strains in both gastrocnemius and soleus muscles were calculated using MRI techniques in healthy human volunteers comparing images taken at static knee angles of $173^{\circ}$ and $150^{\circ}$.

Results Imposed global strains on gastrocnemius were much smaller than local strains. High distributions of strains were encountered, e.g. overall lengthened muscle contains locally lengthened, as well as shortened areas within it. Substantial strains were not limited to gastrocnemius, but were found also in synergistic soleus muscle, despite the latter muscle-tendon complex length remaining isometric (constant ankle angle: i.e. global strain $=0$ ), as it does not cross the knee. Based on results of animal experiments this effect is ascribed to myofascial connections between these synergistic muscles. The most likely pathway is the neurovascular tract within the anterior crural compartment (i.e. the collagen
\end{abstract}

\footnotetext{
P. A. Huijing $(\bowtie)$

Research Institute Move, Faculteit Bewegingswetenschappen,

Vrije Universiteit, Van de Boechorstststraat 9,

1081 BT Amsterdam, The Netherlands

e-mail: P_A_J_B_M_Huijing@ @ fbw.vu.nl

A. Yaman · C. Ozturk · C. A. Yucesoy Institute of Biomedical Engineering, Boğaziçi University, Istanbul, Turkey
}

reinforcements of blood vessels, lymphatics and nerves). However, direct intermuscular transmission of force may also occur via the perimysium shared between the two muscles. Conclusions Global strains imposed on muscle (joint movement) are not good estimators of in vivo local strains within it: differing in magnitude, as well as direction of length change. Substantial mechanical interaction occurs between calf muscles, which is mediated by myofascial force transmission between these synergistic muscles. This confirms conclusions of previous in situ studies in experimental animals and human patients, for in vivo conditions in healthy human subjects.

Keywords m. soleus - m. gastrocnemius - In vivo . Myofascial force transmission - Length change

\section{Introduction}

During the last decade, in situ animal experiments involving unusually minor dissection of muscles (for reviews see [8, 9]) have indicated that, in addition to myotendinous force transmission, transmission of forces also occurs via alternative pathways, between muscles and between muscle and non-muscular tissues. First force is transmitted onto the connective tissue of the stroma of the muscle (possible along the full perimeter along length of muscle fibers), in vivo, there will be several paths available for further transmission, so that not all force will be exerted at the muscle own tendons. The relative stiffness of the paths determining the fraction of force transmitted via them. Via the epimysisum force may be transmitted directly between synergistic muscles, but this may also occur via indirect pathways involving the neurovascular tract (defined as the collagen reinforcements of blood vessels, lymphatics and nerves). It is the 
relative stiffness of neurovascular tracts that make them particularly fit for such transmission. In addition to intermuscular effects also force transmission between muscle and non-muscular structures are expected. Also in this process the neurovascular tract may play an important role, as it is not only connected to muscle, but also via the periost to bones (in casu: tibia and fibula), either directly or via other fascial structures to which it may be connected (such as interosseal membrane, intermuscular septum or even the general fascia).

For humans such transmission has been confirmed by similar in situ experiments performed during an experimental interval of tendon transfer surgery on patients suffering from spastic paresis [13, 14, 20-23]. However, evidence for in vivo occurrence of such so called myofascial force transmission has been limited to, otherwise unexplained, forces exerted by small hand muscles [28].

The application of in vivo non-invasive imaging methods to this problem has been indicated as a potentially promising method (e.g. [10]) to address this question of in vivo importance of myofascial force transmission. Such methods are not only expected to be useful in healthy persons for the in vivo of physiological mechanisms, but also in patients with various afflictions with some muscular involvement. Here, the novel ideas of myofascial force transmission may yield new ways of looking at functional consequences of the pathology.

The purpose of the present work is, as a first step in this process, to test if magnetic resonance imaging methods, can be applied successfully and combined with mechanical engineering methods to test the hypothesis of in vivo myofascial force transmission occurring between synergistic muscles, and if so consider effects of such transmission. One of the features to be expected, if this is found to be the case, is that global strain imposed on the muscletendon complex by joint movement and local strains occurring with muscle will be quite different.

For that purpose, additional work was performed also on legs of human cadavers to quantify global strain on gastrocnemius muscle by movement of the knee, using a novel method of dissection involving the excision of major neurovascular tracts from the leg.

\section{Methods}

Cadaver study: length change of gastrocnemius muscle tendon complex

\section{Materials}

Seven human cadavers ( 3 females, 4 males) were studied. These persons had legally arranged that their bodies were to be donated after death to the Vrije Universiteit for purposes of teaching and research. The cadavers were selected based on availability, but special attention was paid to obtain cadavers of a range of different body sizes. Shortly after death and well prior to the start of this project, the cadavers had been fixed by perfusion of a formaldehyde solution into their left femoral artery and subsequently the whole body was kept in a bath of formaldehyde-based bathing solution for at least 6 months.

\section{Dissection and preparation}

Hemi-pelvectomy was performed on the fixed cadaver (frozen particularly for this purpose to prevent deforming the soft tissues too much) and the whole leg with half of the pelvis still attached was removed from the body and allowed to thaw. From such isolated legs, the skin and subcutaneous fat tissues were removed. After fasciotomy, the crural fascia and fascia lata were removed, exposing the muscles.

Muscles and muscle parts were dissected by severing intermuscular connective tissues. Major blood vessels and nerves and their major branches were removed, including the collagen fiber reinforcement of such neurovascular tracts. These tracts are very stiff even in living limbs, due to their collagen reinforcements and may even become stiffer due to fixation and they limit movement of the muscles in cadavers as a consequence. In addition it should be noted that for living animals these neurovascular tracts are considered as a major path for myofascial force transmission and the inter muscular interaction, that is a consequence of such transmission [8-11, 16, 19].

In order to allow further creation of sufficient movement in the knee joint, both heads of $\mathrm{m}$. gastrocnemius were transected approximately at the middle of their muscle bellies. After dissection, a tube of Bandafix, a wide mesh elastic gauze bandage, was slipped around the leg to hold the muscles together in their original position.

In contrast to previous work with similar purposes [6] and [25], no muscles or parts thereof were removed and the joint capsules were left fully intact. After these preparatory actions, the leg was mounted in the experimental set up (Fig. 1) and the knee angle set to the values required.

The essence of determination of muscle tendon complex length changes after knee angle changes were similar as used in those studies cited above: measuring changes in gap length between transected ends of the muscle with knee angle changes (e.g. Fig. 1c).

Segment length of the lower leg was defined as the perpendicular distance between the lateral aspect of the knee joint cavity and the lateral tip of the lateral malleolus. All measurements were performed by one experimenter to 


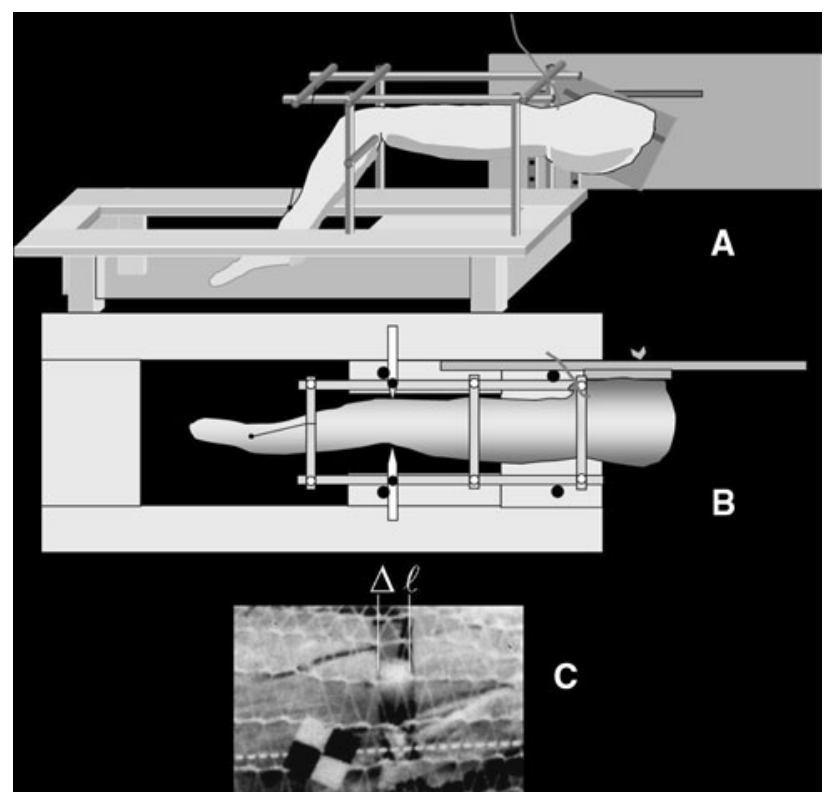

Fig. 1 Schematic of the set up of the cadaver leg experiment. a Lateral view of the leg mounted in the setup. The leg was suspended from a system of bars. Fixing the hip angle, the board attached to the pelvis was attached to a vertical board on the table. b Top view The femur was fixed by pins at the epicondyles. The knee angle could be changed using the string attached to the tibia. $\mathbf{c}$ Photograph of part of a muscle during the experiment. To keep muscles at their original position, the dissected leg was surrounded with an open-weave stocking, still allowing a good view of the muscle under study. The muscle were cut transversely upon which cut ends retracted somewhat $(\Delta l)$. With changes of knee angle, changes of this value from its reference level (i.e. at a knee angle of $135^{\circ}$ ) are a measure of length changes of the muscle tendon complex

avoid interindividual experimenter variation effects on the measurements.

\section{Treatment of data}

All changes in muscle-tendon complex lengths $\left(\Delta l_{\mathrm{m}+\mathrm{t}}\right)$ were calculated as the deviation from the gap value at the reference positions (i.e. a knee angle of $135^{\circ}$, with $180^{\circ}$ representing the fully extended knee) and normalized for segment length.

Such data was least square fitted using the following polynomial equation:

$\Delta l_{\mathrm{m}+\mathrm{t}}=A_{n} \phi^{n}+A_{3} \phi^{3}+A_{2} \phi^{2}+A_{1} \phi+A_{0}$

In Eq. 1, $\phi$ represents the knee joint angle (degrees) and $A_{n}$ to $A_{0}$ are constants determined in the fitting procedure.

Progressively higher order polynomials (maximally sixth order) were fitted to the joint angle length change data of each individual gastrocnemius head, until no statistically significant improvement of the description of the specific data set could be obtained.
MRI analysis

\section{Subjects}

As this part of the experiment was performed in Istanbul, experimental procedures were in strict agreement with the guidelines and regulations concerning human welfare and experimentation set forth by Turkish law, and approved by a Committee on Ethics of Human Experimentation at Boğaziçi University, Istanbul. Five healthy subjects (male, $27 \pm 3$ years old, height $=175 \pm 7 \mathrm{~cm}$ and body mass $=73 \pm 8 \mathrm{~kg}$ ) volunteered for this study. In order to minimize anthropometric differences between subjects, only males were recruited. After a full explanation of the purpose and methodology of the experiments, the subjects gave their informed consent.

\section{Experimental protocol}

During the experiment, each subject was positioned prone in the MRI scanner. The target (left) leg of the subject was brought to a reference position: (1) the ankle angle was fixed at $90^{\circ}$ (Fig. 2) by using an MRI compatible anklefoot orthosis. (2) The knee angle in this prone position with fixed ankle angle (referred to as the undeformed state) was (mean $\pm \mathrm{SD}) 173^{\circ} \pm 3^{\circ}$.

After acquiring sets of 3D high resolution MR images in the undeformed state, the hip and knee joints were brought in flexion by repositioning the upper body: by a support of the chest using MR compatible material: the trunk was elevated until reaching the spatial limits of the MRI machine bore (diameter $=60 \mathrm{~cm}$ ). In this deformed state, the knee angle equaled $150^{\circ} \pm 6^{\circ}$. Subsequently, a second MR image set was collected. Imaging each set took $10 \mathrm{~min}$.

\section{Image acquisition}

3D Turbo Flash based MR coronal image sets were collected using a 3T Siemens Trio scanner with 6-channel cardiac array coil (matrix size $=320 \times 320 \times 144$, voxel size $=0.8 \times 0.8 \times 0.8 \mathrm{~mm}, \mathrm{TR}=2,000 \mathrm{~ms}, \mathrm{TE}=3.94$ $\mathrm{ms}$, no fat suppression, flip angle $=12^{\circ}$, bandwidth $=130 \mathrm{~Hz}$ per pixel). The region between the most proximal part of the head of the fibula and the most proximal location of the transverse crural ligament was imaged.

\section{Calculation of strain fields}

In order to align images of the deformed and undeformed states as well as possible, Demons algorithm [24] an intensity based non-rigid non-parametric image analysis technique, was applied to the MR image sets. Demons 


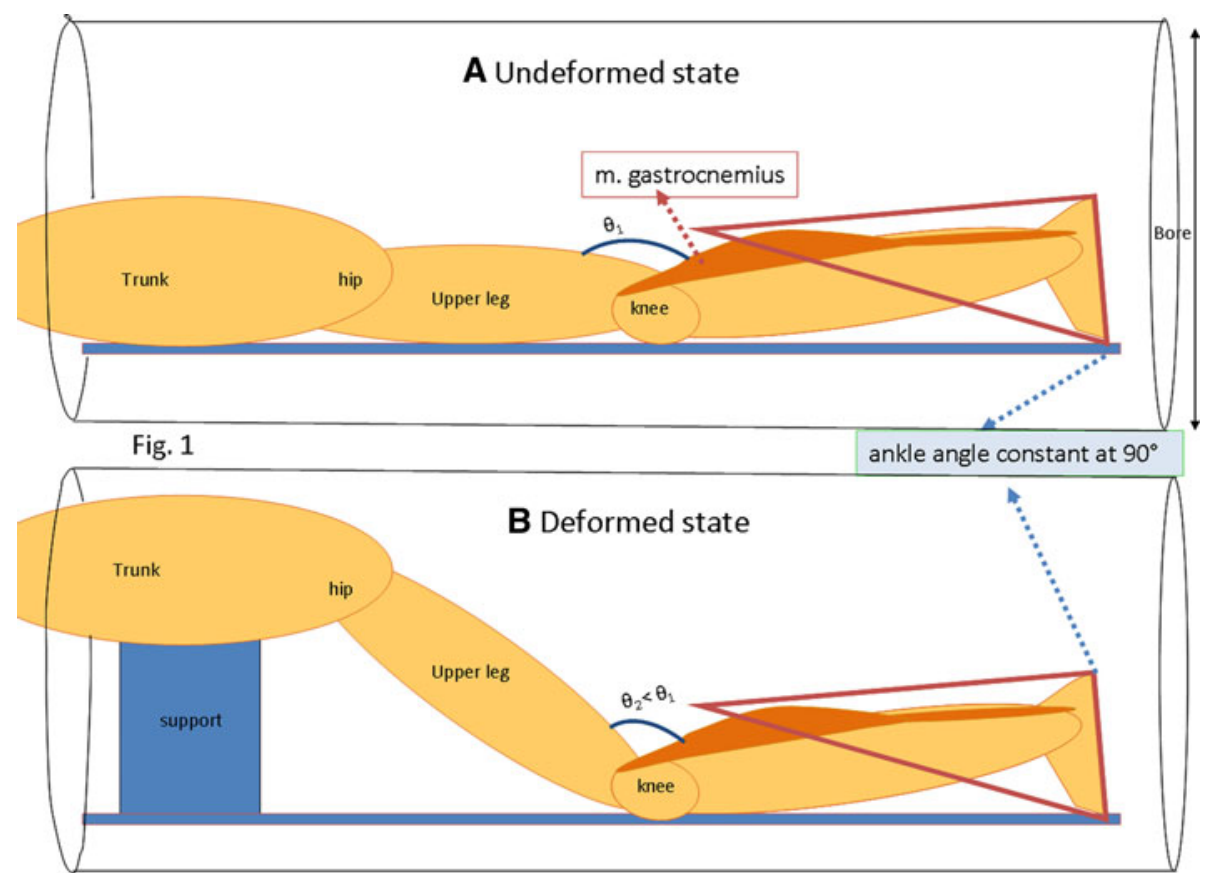

Fig. 2 Schematic representation of the experimental setup within the MRI machine. The subject is placed in prone position within the bore of the machine. An MRI compatible ankle-foot orthosis (triangle) is applied to fix ankle joint angle at $90^{\circ}$. A small space between posterior side or the lower leg and ankle-foot orthosis and also between anterior side of the lower leg and MR table is allowed to

algorithm has been improved by several authors (a) to prevent instabilities for small image gradient values by additional constants [4], (b) to extend the algorithm using optimizers (i.e. gradient descent 4) and limited use of memory [15], (c) to adapt for large deformations by multiresolution approach and (d) to accelerate by extending the equation using also the image gradients at the deformed state [26]. Note that, in our present study a version including these improvements of Demons algorithm was used.

After alignment of images, information on real deformation is contained in minimized image differences. Simultaneously with alignment process, the algorithm recalculates the exact cubic shape made up of four original voxels from the undeformed state into a deformed shape of approximately constant volume, again by an iterative process, while still trying to fit undeformed and deformed states. The volume elements of the deformed shapes corresponding to the original undeformed elements are assigned using three criteria: (1) the arrays of voxel gray scale values, (2) their array of grey scale differences (i.e. difference images), and (3) their grey scale gradients within the images. During each iteration performed, the displacement fields are smoothed by a Gaussian kernel for regularization of global motion. avoid exertion of other external forces. a Condition arbitrarily defined as the undeformed state, with knee joint angle at $\approx 173^{\circ}$. b Condition arbitrarily defined as the undeformed state, with knee joint angle at $\approx 150^{\circ}$. This condition was attained by placing a support (rectangle) under the trunk which changed hip and knee joint angles, but left the position of the lower leg unchanged

To calculate the strain tensor, the deformation gradient matrix, $F$ was calculated first by using displacement gradient tensor $(\nabla u)$ in material coordinates:

$F=\nabla u+I$

Green-Lagrange strain tensor, $E$ was calculated as:

$E=\frac{1}{2}\left[F^{T} F-I\right]$

This tensor is then rotated, to remove shear effects, and calculate principle strains. The first and third principle strains were used separately in the analyses, since they represent local lengthening and shortening, respectively.

\section{Estimation of errors introduced by applying demons algorithm}

Image sets for each subject of the undeformed state were transformed by a "rigid body motion". This means that no real deformation was introduced. The rigid body motion imposed on the data consisted of (1) a $10^{\circ}$ rotation within the cross-sectional plane corresponding to an endorotation of knee that may be expected as the knee flexed [18] and any global movement that may occur when changing the 
knee angle as described. (2) A $3^{\circ}$ rotation in the coronal plane, (3) a $3^{\circ}$ rotation in the sagittal plane and (4) a $4 \mathrm{~mm}$ translation in the axial direction. Since in reality, the position of lower leg was kept constant, rotations in cross-sectional and sagittal planes and the translation in axial directions should be infinitesimal. The original and transformed image sets were then aligned and compared as described above and principle strains were calculated. Note that, since in reality, the position of lower leg was kept constant, rotations in cross-sectional and sagittal planes and the translation in axial directions should be infinitesimal. Therefore, the rigid body motion imposed representing a much larger scale motion than caused by moving the knee joint allows a fairly critical testing of artifacts during strain calculations, since by definition such motion should cause no deformation. Therefore, deviation of calculated principle strains from zero after rigid body image rotation are used as estimates of errors in strain calculations.

\section{Further treatment of data}

In order to assess tissue effects of changing knee angle, a slice group consisting of 30 consecutive cross-sectional slices was selected manually for each subject (Fig. 2a): the most proximal slice of the group was located at the upper third of the length of imaged portion of the lower leg in a longitudinal image (a level corresponding approximately to the mid-belly of $\mathrm{m}$. gastrocnemius).

For each slice analyzed, anatomical regions representing muscles or compartments (i.e. muscle groups) were distinguished manually by outlining their boundaries. Doing so, m. gastrocnemius, m. soleus, deep flexor muscles, were marked (Fig. 2b).

Effects of changing the knee angle on deformations within passive lower leg muscles were assessed per marked area: Note that due to the fixed ankle angle, the muscle tendon complexes of lower leg muscles not crossing the knee (all except $\mathrm{m}$. gastrocnemius, i.e. m. soleus, deep flexors, peroneal and anterior crural muscles) remain isometric. For each anatomical region separately, mean $\pm \mathrm{SE}$ of principle strains were calculated occurring due to (1) the knee angle change and (2) errors introduced by the algorithm.

\section{Statistics}

For each anatomical region separately, sample of principle strains and error strains were compared. Since the calculated principle strains and error strains are, by definition, not distributed normally, nonparametric Wilcoxon rank sum tests $(p<0.05)$ were used to test for significant differences.
If principle strains were significantly bigger than error strains, we concluded that the effect should be assigned to altered body position (most important: knee joint angle).

\section{Results}

Global strain in gastrocnemius muscle as a consequence of knee movement

As human gastrocnemius muscle functions as a knee flexor, changes in knee joint angle will lead to changes of muscletendon complex length (lengthening for knee extension, shortening for knee flexion), the size of which is determined by actual moment arm length and actual knee movement imposed. Figure 3 shows results measured in human cadavers for a sizable range in knee joint angles.

To facilitate further use of the fitted curves, the values their coefficients selected in the fitting process are shown in Table 1. Note that knee angle length change curves were statistically best explained by second order polynomials (A3 being equal to zero), explaining the linear nature of the knee angle moment curves (as obtained by differentiation).

The data show that gastrocnemius moment arms at the knee are not constant, but increase with knee flexion.
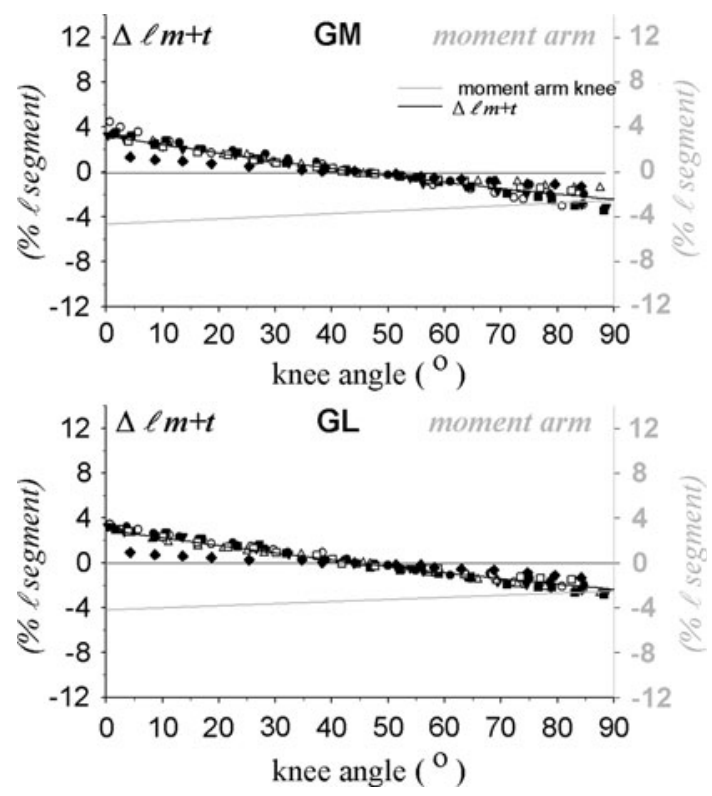

Fig. 3 Gastrocnemius muscle-tendon complex length changes as a function of knee angle. Upper panel $\mathrm{m}$. gastrocnemius caput medialis (GM). Lower panel m. gastrocnemius caput lateralis (GL). Length change $(\Delta l \mathrm{~m}+\mathrm{t}$, use left $Y$-axis $)$ is plotted as individual $(n=7)$ data and normalized for tibia length ( $l$ segment) A polynomial is fitted to the data (drawn black line, use left $Y$-axis). The gray lines (use right $Y$-axis) describe the relation of moment arm and knee joint angle, as calculated from the fitted knee angle-length change curve by differentiation. $180^{\circ}$ represents a fully extended knee 
Table 1 Polynomial coefficients obtained by fitting pooled joint angle-normalized length change data sets for each muscle head

\begin{tabular}{llllll}
\hline Joint & Muscle head & $A_{3}$ & $A_{2}$ & $A_{1}$ & $A_{0}$ \\
\hline Knee & GM & 0 & 0.000206 & -0.081560 & 3.240837 \\
Knee & GL & 0 & 0.000163 & -0.073618 & 2.951952 \\
\hline
\end{tabular}

Polynomial: $\Delta l_{\mathrm{m}+\mathrm{t}}=A_{3} \phi^{3}+A_{2} \phi^{2}+A_{1} \phi+A_{0}$, fitted to knee anglegastrocnemius length change data (shown in Fig. 3), where $\phi$ represents knee joint angle, defined according to the conventions described in the methods

Differences between medial and lateral heads of the gastrocnemius in both length change moment arm are quite small. For the much smaller range of knee joint angles to be considered below in human subjects using MRI analysis the values of length change normalized for tibia length amounted to only approximately $1.5 \%$ of tibia length. As gastrocnemius muscle-tendon complex is somewhat longer than tibia length, expected imposed global strain due to length changes of the muscle tendon complex will be somewhat less than $1.5 \%$.

Within the context of this experiment (constant ankle joint angle, i.e. a globally isometric soleus muscle), length changes of gastrocnemius muscle with changing knee angles are also indicative for the relative movements of soleus and gastrocnemius muscles. This is important as the relative movement between muscles will co-determine stiffness of the connections between them.

\section{MRI analysis of local muscle strain}

\section{Accuracy in displacement and local strain calculations}

For the muscular regions studied, error strains calculated are small (mean $<1.5 \%$;) Therefore, it is concluded that the image handling methods applied successfully aligns images and does not introduce major errors or artifacts in the calculation of principle strains for gastrocnemius and soleus muscles.

\section{Effects of knee angle change on in vivo passive muscular strains}

Figure 4 shows a typical example of a comparison of deformation of a regular grid mapped on a sample's most proximal slice in the undeformed state (Fig. 3b) and the deformed grid calculated for the deformed state (Fig. 3c). Note that, for clarity, this example (Fig. 3b) presents 2-Dresults, while a $3 \mathrm{D}$ analysis was performed. Note also that grid deformations are encountered in subcutaneous fat tissues and skin, as well as in muscle tissue.

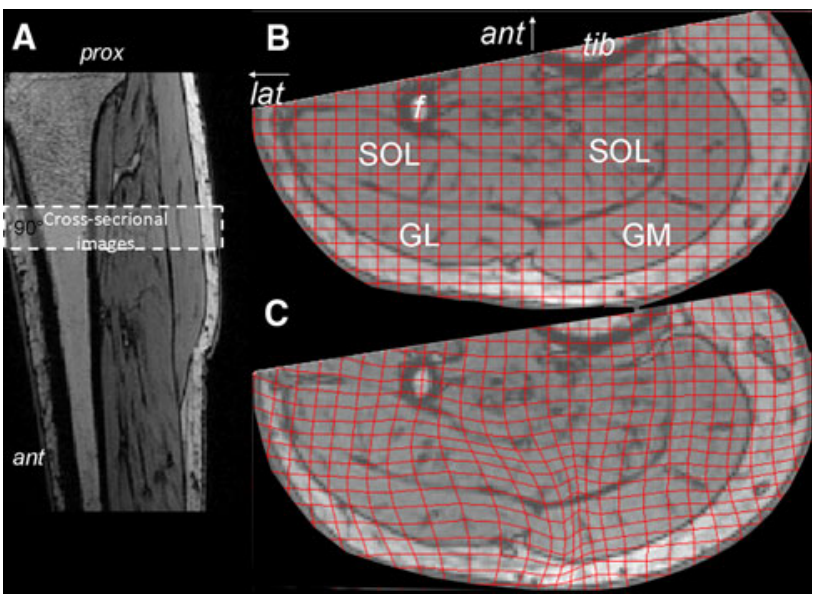

Fig. 4 Typical example of MRI images with superimposed undeformed and deformed grids. a Longitudinal image of the lower leg, indicating the location of the cross-sectional images taken. b Crosssectional MRI image of the lower leg (most proximal image of the stack) in the undeformed state, with the undeformed grid imposed and indications of anatomical structures to be seen. Knee joint angle $\approx 173$ Ankle joint angle $=90^{\circ}$. c Cross-sectional MRI image of the lower leg (most proximal image of the stack) in the deformed state, with the deformed grid imposed (Knee joint angle $\approx 150^{\circ}$. Ankle joint angle $=90^{\circ}$ ). "Ant" indicates anterior side or direction (arrow) and "lat" indicates lateral side and direction (arrow "Tib" and " $f$ " indicate tibia and fibula, respectively, and the relevant muscles are indicated as follows: $G L$ and $G M$ represent m. gastrocnemius caput lateralis and medialis, respectively, and $S O L$ indicates $\mathrm{m}$. soleus

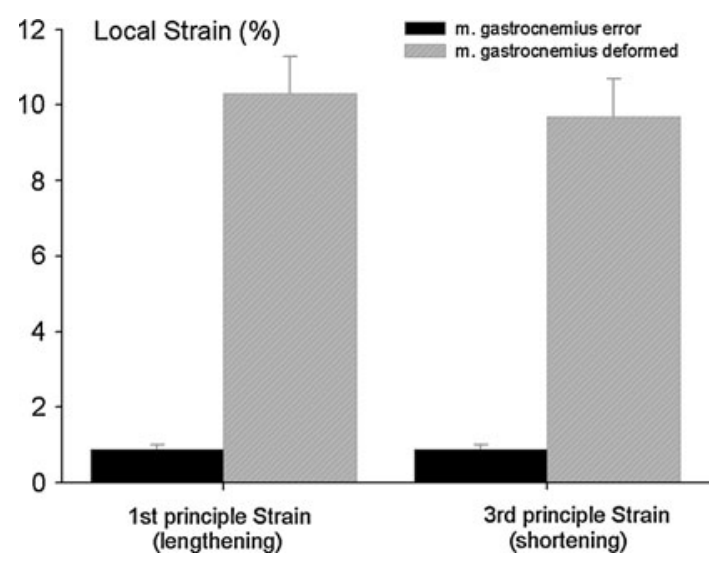

Fig. 5 Mean principle local strains within $m$. gastrocnemius. The error strains (dark bars) were calculated after large scale solid body rotation of the images, i.e. involving no actual deformation of tissues. Principle strains calculated (hatched bars) by comparing images taken at knee joint angles equal to $173^{\circ} \pm 3^{\circ}$ and $150^{\circ} \pm 6^{\circ}$, respectively. Note that ankle joint angle remained at $90^{\circ}$ while collecting both image sets. Mean values and SE are plotted

\section{Within m. gastrocnemius}

Mean principle strains within $\mathrm{m}$. gastrocnemius are significantly higher than error strains for this muscle (Fig. 5). Being a knee flexor for $\mathrm{m}$. gastrocnemius, knee extension 
corresponds to overall lengthening strain and knee flexion to overall shortening strain. Mean local shortening in $\mathrm{m}$. gastrocnemius muscle remained limited to values $\approx 10 \%$ ), but note that similar values were attained for local lengthening. This indicates the presence of considerable strain distributions within this muscle. In addition, note that such peak values are much bigger than the global strain imposed on the whole muscle-tendon complex.

\section{Within m. soleus}

It should be noted that if soleus and gastrocnemius muscle would be fully independent mechanically, no local strains should be encountered within soleus muscle, since this muscle-tendon complex does not cross the knee joint and as consequence, no global strains are imposed on this muscle-tendon complex. In contrast to such expectation, mean principle strains for $\mathrm{m}$. soleus are also significantly different from error strain values and attain a considerable magnitude (between 8.5 and $9.4 \%$ for shortening and lengthening, respectively, Fig. 6).

Therefore, we conclude that considerable mechanical interaction between these synergistic plantar flexor muscles accompanies altered knee joint angles. Such local strains are very hard to explain, unless intimate connections between these synergistic muscles are assumed that transmit force between gastrocnemius having been exposed to length changes, with soleus muscle remaining at an overall intermediate muscle-tendon complex length (Fig. 7).

It also concluded that the pattern of strains is not a simple one: First, considerable variation between individuals was encountered. Some of this was also seen for global strain measurements for m. gastrocnemius (Fig. 3) However, variation in local strains is much higher (Fig. 5). It

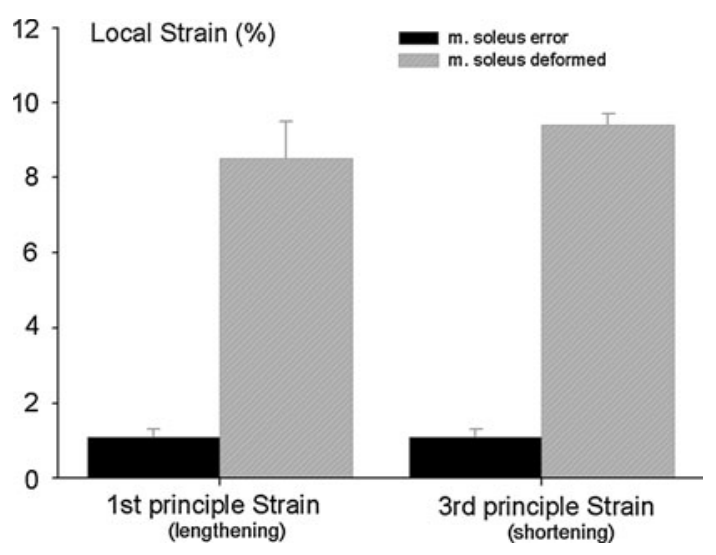

Fig. 6 Principle strains within $\mathrm{m}$. soleus. The error strains (dark bars) were calculated after large scale solid body rotation of the images, i.e. involving no actual deformation of tissues. The strains calculated (hatched bars) by comparing images taken at knee joint angles equal to $173^{\circ} \pm 3^{\circ}$ and $150^{\circ} \pm 6^{\circ}$, respectively. Note that ankle joint angle remained at $90^{\circ}$ while collecting both images

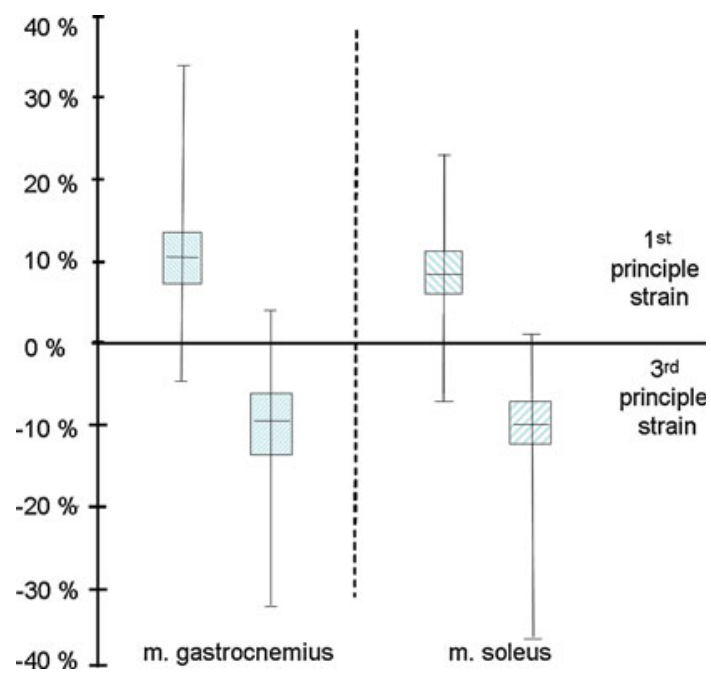

Fig. 7 Effect of altered knee angle: local shortening and lengthening effects. The horizontal line inside the box represents the median strain value; the upper and lower edges of a box itself represent upper and lower quartiles (i.e., the 75th and 25th percentiles), respectively, and lines extending from the median represent range of values of the principle strain plotted. Data are analyzed across all subjects $(n=5)$

should, however, be remembered that these values contain individual sources of variation, as well as variation per location within muscles. Second, at different locations within soleus muscle local lengthening will occur simultaneously with local shortening at other locations.

\section{Discussion}

Major results of the present work are the findings that (1) local strains within the muscle are very much higher that the global strains imposed on it (2) such local strains are not limited to the specific muscle that is exposed to length changes, but also occur within synergistic muscle kept at constant muscle-tendon complex length (global isometric condition).

This means that, also in vivo, synergistic muscle are not mechanically independent. This idea is compatible with many results of animal experiments (for reviews see [8, 9]), on the basis of which the ideas of epimuscular myofascial force transmission were developed over the last decade.

It should be noted that based on such animal experiments myofascial force transmission between antagonistic muscles is also predicted. Presently, further analysis of the image data set used in this study is progress to test that hypothesis. A preliminary analysis indicates that such a hypothesis is likely to be accepted [27].

The essence of the concept of myofascial force transmission is that force transmission of muscle is not limited to that between a specific muscle and its own tendon(s), but that simultaneously a fraction of the force generated 
(depending on conditions, sometimes quite considerable) is transmitted "sideways" between muscles, either directly between adjacent muscle bellies (intermuscular transmission)via the shared epimysium between the muscles, or via extramuscular connective tissues linking synergistic muscles and between muscle and bone (extramuscular force transmission). In fact, such concepts involve the notion of continuity of tissues within a limb segment that certainly has been considered in history (for a short historical review see [9]). In addition to that it seems that one of the first to fully ascribe this notion of continuity of connective tissues was the Dutch anatomist Andreas Bonn, who in his doctoral dissertation [2] reproduced in [3], laid a solid base for such ideas of continuity of connective tissues, even though he did not focus specifically on muscular connections. Despite the early work, until recently the connective tissue matrix was either ignored or considered a benign scaffold keeping things together. Therefore, further going functional consequences were not considered. The resent work indicates that the concept of tissue continuity needs to be revived as it also leads to important functional effects, such as muscular interaction by myofascial force transmission, also occurring in vivo in human subjects.

The concept of continuity of tissues within limbs

The anatomical substrate of the different types of myofascial force transmission can be described as follows: the sarcomeres within muscle fibers are connected to the intracellular cytoskeleton that is connected via subsarcolemmal actin filaments (differing from the sarcomeric ones) to trans-sarcolemmal molecules (integrins and/or sarcoglycans) that is connected to the endomysium, a connective tissue tube within each muscle fiber is located. The collection of endomysial tubes of a muscle fascicle form a continuous system that is surrounded by another connective tissue tube, the (primary) perimysium. Groups of small fascicles or bundles of muscle fibers are surrounded as well by epimysial tubes (secondary ones). In turn, the collection of perimysia constitutes a continuous system, sometimes called a stroma that is delimited by the epimysium. In vivo muscle is working within its natural context of connective tissues. For synergistic muscles, walls of epimysial tubes are shared, except for places where neurovascular tracts passes between muscles. However, neurovascular tracts also connect muscles to each other as branches containing vessels and nerves pass into the muscle and are continuous with the tertiary perimysium that does not fully surrounds fascicles or groups of small fascicles. The neurovascular tracts are attached also to the periost surrounding the bones and to other nonmuscular elements of the connective tissues system, such as the interosseal membrane, the intermuscular septum which themselves are continuous with the general fascia of the limb. This describes the full continuity of the system. However, if myofascial force transmission will occur to an appreciable extent using a specific pathway, is not so much determined by the fact that it is connected, but rather the actual stiffness of the connections, which depends on the actual circumstances such muscle length (i.e. joint angle) and relative positions of muscles with respect to each other and to the non-muscular elements described above. Our present results are indicative that at least some of the pathways mentioned are stiff enough to create substantial local length changes within soleus muscle. The specific identification of such pathways should be pursued in future work.

Myofascial force transmission with imposed joint position and joint movement

It should be noted that all previous physiological and surgical experiments proving epimuscular myofascial force transmission involved length changes imposed directly on the muscle (by moving the proximal or distal tendon) without simultaneous movement of the joint. It is very easy to show that the extramuscular neurovascular tracts (i.e. the collagen reinforcement of blood and lymph vessels and nerves) is exposed to considerable changes of length, position and tension (for an informal way of showing that see [8]). This means that in vivo myofascial force transmission could potentially quite different in effects or size if such joint movement were present as well.

Recently, Maas and Sandercock acted on such a potential limitation of previous work and performed an elegant experiment in the cat. Their experiment [17] involved features that resemble those also of our present study: Ankle angle was kept constant, while the knee was moved and force exerted by soleus muscle was measured and net ankle joint moment was calculated on the basis of externally measured forces at the foot. With soleus muscle in its natural position, they did not find any sign of intermuscular interaction. However, if soleus was moved from its original length and position (corresponding to the imposed constant ankle angle), myofascial effects were apparent immediately. Based on these results, they generalized their findings by concluding that mechanical interaction between muscles does not occur under physiological circumstances in vivo. In reaction, Herbert and co-workers pointed out Maas and Sandercocks experiment was not really an in vivo experiment [7] indicating the need for such real in vivo work. It is clear that given our present results Maas and Sandercocks generalized conclusion is not tenable.

This is supported also by recent work [1] applying in vivo ultrasound imaging techniques on human triceps 
surae. Selective stimulation of medial gastrocnemius muscle, as well as passive knee extension yielded displacement of both medial gastrocnemius and soleus muscles.

Reasons for such contrasting results are not immediately apparent. It could be related to differences of species or to differences in dynamics: In the present experiment, we had to compare two static situations (due to long imaging times), while Maas and Sandercock imposed joint movement dynamically. Therefore, there is a need for direct experimental comparison of effects such static and dynamic conditions.

Further limitations of our present experiment

In addition to some limitations discussed above, we need to consider some others.

\section{Strain in muscle fiber direction}

It should be kept in mind that the strains reported in the present work are local muscle strains, but expressed in terms of an outside coordinate system.

Particularly in human gastrocnemius and soleus muscles this is a real limitation, since both are very pennate (as is apparent also from the huge difference between fascicle length and muscle length). In both muscles, they have to span only the oblique distance between proximal and distal aponeurosis which is in the order of 5 and $3 \mathrm{~cm}$, respectively. Gastrocnemius muscle has a unipennate architecture and soleus muscle a bipennate structure indicative of quite different muscle fiber directions at opposite sides of the distal aponeurosis. Myofascial force transmission takes place at the interface between the ends of the muscle fibers and extracellular collagen fibers inserted into the myotendinous junction that are collected to form the collagen bundles that run predominately in the longitudinal direction of the aponeurosis.

The physiologically important variable is of course local strain in the direction of muscle fibers, representing length changes of sarcomeres. In previous finite element modeling studies, due to epimuscular myofascial force transmission major strain heterogeneity in the local muscle fiber direction is a common feature [29-33]. Our present results suggest that sizable strains in the local muscle fiber direction (but somewhat smaller than the peak strains reported presently) are expected also in human subjects in vivo. However, this needs to be confirmed. Fortunately, MRI-imaging is also capable to provide techniques to estimate fiber directions locally within the muscle.

On the other hand, the present findings show local deformations involving lengthening and shortening occurring simultaneously at different locations within the same muscle and can differ from in size as well as direction from the global strains imposed on the muscle. Model studies also indicate that (e.g., for a muscle at high length), a presence within the same muscle fiber of not only lengthened sarcomeres, but also considerably shortened ones are to be expected (e.g. [27]). If sarcomere length heterogeneity to such a major extent occurs also in vivo, needs to be tested. To do that, work is in progress applying additional MRI methods to determine the direction of muscle fibers locally within the collected images and convert present strains results to local muscle fiber strain in fiber and crossfiber directions.

\section{Muscle activity}

Since it is difficult to combine electromyography with the high magnetic field within the bore of the MRI machine, we have had no rigid control of activity of target muscles. Measurements performed outside of the MRI machine indicate that the target muscles may not have been fully passive, but activities are expected to have relatively low $(<0.5 \% \mathrm{MVC})$. Temporal variation of muscle activity is unlikely to have contributed to the results because of long imaging times. In addition, differences in muscle activity could not cause simultaneous occurring differences of first principle strain (indicative of lengthening) and third principle strains (indicative of shortening).

Consideration of some potential clinical implications of the concept of myofascial force transmission

The concepts of epimuscular myofascial force transmission should have major effects on our thinking of the physiology and pathology of muscular function, within such altered views also adaptation of clinical concepts are expected and are only just beginning to appear.

In surgery, it is important to realize that dissection necessary to gain entrance to a limb will lead to a generally higher compliance of the continuous fascial or connective tissues system, unless an extremely small window within the general fascia is used to gain access. This means that in most surgery, the properties of this system and thus also of muscles are changed before the surgeon may feel that the actual intervention has really started. It is likely that preventing or limiting such altered properties constitutes a considerable advantage of minimal invasive surgery.

Regarding spastic paresis, where relative positions of muscles under spastic neural control are altered with respect to non-spastic muscle, as well as non-muscular structures because spastic muscle will have a tendency to be shorter than non spastic muscles, the knowledge of epimuscular myofascial force transmission may provide a new way of thinking in trying to explain the nature of the 
relatively fixed preferential positions of joints crossed (sometimes referred to as contracture). Recently, we have presented a hypothesis [8] involving interaction between antagonistic muscles due to epimuscular myofascial force transmission between non-spastic and spastic muscle. It is clear that that stiffness of elements of epi- and intramuscular myofascial links needs to be considered. Presently, our group has, evidence [5, article in preparation] that that the tertiary epimysium is thickened (and presumably stiffer) in human spastic flexor carpi ulnaris muscle (FCU). This fits with earlier findings of our group [22] that after partial dissection (for the purpose of subsequent tendon transfer to an extensor insertion) the length force characteristics of FCU in human patients measured during the operation did not give any indication that could explain the preferential wrist position of these patients. This indicates that not the muscle characteristics per se are affected as secondary effects of the spastic neural control, but rather the mechanical interaction with its environment leading to different functionalities. Particularly for spastic human triceps muscle, one may expect such effects at much higher level due to its relatively high moment arm with respect to and talo-crural and subtalar joints and their effects on relative position of this muscle group.

Based on the hypothesis described, rather than performing tendon transfers, the surgeon may consider looking for ways to interrupt the mechanical interaction between antagonistic muscles. However, this is not expected to be easy, since it seems not likely that the mechanical role of the neurovascular tract can be interfered with, and if one could, to make this effect permanent (prevent regeneration of intermuscular mechanical linkages).

Our knowledge of myofascial force transmission make it intuitive why genetic deficiencies of proteins that are a part of the trans-sarcolemmal chain of molecules leads to serious disease (muscular dystrophies). However, much more work is needed to enhance our understanding of the mechanics of these phenomena. We have some animal experimental work in progress on this subject.

Another example is recent work on tenascin-X deficient serving as a model for Ehlers-Danlos disease and wild type mice [12]. For this disease, clinically, changes in muscle function are not considered often, since standard histological tests applied do not indicate muscle pathology. Nevertheless, the interaction of muscles with much more compliant connective tissues within limb segments in such patients are likely to contribute to altered muscle characteristics with functional consequences. If coordination of muscular activity by the central nervous system is not arranged to deal with this altered condition optimally, it could well explain patients complaint regarding fatigue.
It is clear that more in vivo MRI analysis in patients with one of several diseases is indicated. Methods similar to the ones described here may help to do that.

Acknowledgments The MRI part of this study was supported by (1) the Turkish State Planning Organization (DPT) under the TAM Project (2007K120610) and Life Sciences Research Center Project (2009K120520), (2) The Scientific and Technological Research Council of Turkey (TÜBITAK) under grant 107E274 and (3) The Turkish Academy of Sciences (TÜBA) under Distinguished Young Scientist Award to Can A. Yucesoy. Technical support of imaging aspects of the project by Guus C. Baan at the Faculteit Bewegingswetenschappen, Vrije Universiteit, Amsterdam is gratefully acknowledged. For the cadaver part of this study, Ulricke M. Althusius and Jorik and H.W.C. van Straten, at the time, Master students at the Faculteit Bewegingswetenschappen, Vrije Universiteit, Amsterdam, provided excellent technical support in partial fulfillment of degree requirements. Ethical standards The experiments reported comply with the current laws of the country in which they were performed (i.e. The Netherlands and Turkey, respectively).

\section{Conflict of interest None.}

Open Access This article is distributed under the terms of the Creative Commons Attribution Noncommercial License which permits any noncommercial use, distribution, and reproduction in any medium, provided the original author(s) and source are credited.

\section{References}

1. Bojsen-Møller J, Schwartz S, Kalliokoski KK, Finni T, Magnusson SP (2010) Intermuscular force transmission between human plantar flexor muscles in vivo. J Appl Physiol 109(6): 1608-1618

2. Bonn A (1763) Specimen Anatomico-Medicum Inaugurate de Continuationibus Membranarum, quod publico; ac solemni disquisitioni submisit, Andreas Bonn, Amsteloedamo-Batavus ad diem 14 Octobris 1763. Wishoff et Haak, Lugdunum Batavorum

3. Bonn A (1869) De continuationibus membranarum. In: Sandifort E (ed) Thesaurus dissertationum, programmatum, aliorumque opusculorum selectissimorum. Volumen secundum. apud Henricum Beman, Roterodami, pp 265-303 \& Corollaria \& Tabula V

4. Cachier P, Pennec X, Ayache N (1999) Fast non-rigid matching by gradient descent: study and improvement of the 'Demons' algorithm INRIA. Research Report 3706, Institut National de Recherche en Informatique et en automatique (INRIA), France, June 1999. http://www-sop.inria.fr/asclepios/biblio/Year/1999. complete.html\#Cachier:RR:99

5. De Bruin M, Smeulders MJ, Kreulen M, Huijing PA, Jaspers RT (2010) A Comparison of Intramuscular Connective Tissue Content in Spastic Muscle versus Healthy Controls. In: [s.n.] Conference proceedings of the 11th Triennial Congress of the International Federation of Societies for Surgery of the Hand. Seoul, South Korea, pp 256-257

6. Grieve DW, Pheasant S, Cavanagh PR (1978) Prediction of gastrocnemius length from knee and ankle joint posture. In: Asmussen E, Jørgensen K (eds) Biomechanics VI, vol A. University Park Press, Baltimore, pp 405-412

7. Herbert RD, Hoang D, Gandevia SC (2008) Are muscles mechanically independent? J Appl Physiol 104:1549-1550

8. Huijing PA (2007) Epimuscular myofascial force transmission between antagonistic and synergistic muscles can explain 
movement limitation in spastic paresis. J Electromyogr Kinesiol 17:708-724

9. Huijing PA (2009) Epimuscular myofascial force transmission: a historical review and implications for new research. International society of biomechanics Muybridge award lecture, Taipei, 2007. J Biomech 42:9-21

10. Huijing PA, Baan GC (2001) Extramuscular myofascial force transmission within the rat anterior tibial compartment: proximodistal differences in muscle force. Acta Physiol Scand 173:1-15

11. Huijing PA, Baan GC (2001) Myofascial force transmission causes interaction between adjacent muscles and connective tissue: effects of blunt dissection and compartmental fasciotomy on length force characteristics of rat extensor digitorum longus muscle. Arch Physiol Biochem 109:97-109

12. Huijing PA, Voermans NC, Baan GC, Busé TE, Engelen BGM, de Haan A (2010) Muscle characteristics and altered myofascial force transmission in tenascin-X-deficient mice, a mouse model of Ehlers-Danlos syndrome. J Appl Physiol 109:986-995

13. Kreulen M, Smeulders MJ, Hage JJ, Huijing PA (2003) Biomechanical effects of dissecting flexor carpi ulnaris. J Bone Joint Surg Br 85(6):856-859

14. Kreulen M, Smeulders MJC, Hage JJ, Huijing PA (2002) Biomechanical aspects of surgical muscle dissection in a tendon transfer procedure. J Hand Surg 27B(Suppl.1):55

15. Kroon DJ, Slump CH (2009) MRI modalitiy transformation in demon registration. In: Proceedings of the Sixth IEEE international conference on symposium on biomedical imaging Boston, Massachusetts. IEEE Press, Piscataway, pp 963-966

16. Maas H, Meijer HJM, Huijing PA (2005) Intermuscular interaction between synergists in rat originates from both intermuscular and extramuscular myofascial force transmission. Cells Tissues Organs 181:38-50

17. Maas H, Sandercock TG (2008) Are skeletal muscles independent actuators? Force transmission from soleus muscle in the cat. J Appl Physiol 104(6):1557-1567

18. Moglo KE, Shirazi-Adl A (2005) Cruciate coupling and screwhome mechanism in passive knee joint during extension-flexion. J Biomech 38:1075-1083

19. Rijkelijkhuizen JM, Baan GC, de Haan A, de Ruiter CJ, Huijing PA (2005) Extramuscular myofascial force transmission for in situ rat medial gastrocnemius and plantaris muscles in progressive stages of dissection. J Exp Biol 208:129-140

20. Smeulders MJ, Kreulen M, Hage JJ, Baan GC, Huijing PA (2002) Progressive surgical dissection for tendon transposition affects length-force characteristics of rat flexor carpi ulnaris muscle. J Orthop Res 20:863-868

21. Smeulders MJ, Kreulen M, Hage JJ, Huijing PA, van der Horst CM (2004) Intraoperative measurement of force-length relationship of human forearm muscle. Clin Orthop 418:237-241
22. Smeulders MJ, Kreulen M, Hage JJ, Huijing PA, van der Horst CM (2004) Overstretching of sarcomeres may not cause cerebral palsy muscle contracture. J Orthop Res 22:1331-1335

23. Smeulders MJ, Kreulen M, Hage JJ, Huijing PA, van der Horst CM (2005) Spastic muscle properties are affected by length changes of adjacent structures. Muscle Nerve 32:208-215

24. Thirion JP (1998) Image matching as a diffusion process: an analogy with Maxwell's demons. Med Image Anal 2:243-260

25. Visser JJ, Hoogkamer JE, Bobbert MF, Huijing PA (1990) Length and moment arm of human leg muscles as a function of knee angles. Eur J Appl Physiol 61:453-460

26. Wang H, Dong L, O'Daniel J, Mohan R, Garden AS, Ang KK, Kuban DA, Bonnen M, Chang JY, Cheung R (2005) Validation of an accelerated 'demons' algorithm for deformable image registration in radiation therapy. Phys Med Biol 50:2887-2905

27. Yaman A, Ledesma-Carbayo M, Baan GC, Huijing PA, Ozturk C, Yucesoy CA (2009) Assessment using MRI shows that intersynergistic as well as inter-antagonistic epimuscular myofascial force transmission has sizable effects within the entire human lower leg, in vivo. In: Huijing PA, Hollander P, Findley TW, Schleip R (eds) Fascia research II. Basic science and implications for conventional and complementary health care. Elsevier, München, pp 147-148

28. Yu WS, Kilbreath SL, Fitzpatrick RC, Gandevia SC (2007) Thumb and finger forces produced by motor units in the long flexor of the human thumb. J Physiol Lond 583:1145-1154

29. Yucesoy CA, Huijing PA (2007) Substantial effects of epimuscular myofascial force transmission on muscular mechanics have major implications on spastic muscle and remedial surgery. J Electromyogr Kinesiol 17:664-679

30. Yucesoy CA, Koopman BH, Baan GC, Grootenboer HJ, Huijing PA (2003) Effects of inter- and extramuscular myofascial force transmission on adjacent synergistic muscles: assessment by experiments and finite-element modeling. J Biomech 36(12): $1797-1811$

31. Yucesoy CA, Koopman HFJM, Baan GC, Grootenboer HJ, Huijing PA (2003) Extramuscular myofascial force transmission: experiments and finite element modeling. Arch Physiol Biochem 111(4):377-388

32. Yucesoy CA, Koopman HFJM, Grootenboer HJ, Huijing PA (2003) Effects of inter- and extramuscular myofascial force transmission on adjacent synergistic muscles: assessment by experiments and finite-element modeling. J Biomech 36:17971811

33. Yucesoy CA, Maas H, Koopman BH, Grootenboer HJ, Huijing PA (2006) Mechanisms causing effects of muscle position on proximo-distal muscle force differences in extra-muscular myofascial force transmission. Med Engin Phys 28:214-226 